Research Article

\title{
Comparison of Methodologies for the Detection of Multiple Failures Using Acoustic Images in Fan Matrices
}

\author{
Lara del Val (D, ${ }^{1}$ Alberto Izquierdo, ${ }^{2}$ Juan J. Villacorta, ${ }^{2}$ and Luis Suárez ${ }^{3}$ \\ ${ }^{1}$ Mechanical Engineering Department, School of Industrial Engineering, University of Valladolid, Valladolid 47011, Spain \\ ${ }^{2}$ Signal Theory and Communication Systems, and Telematics Engineering Department, \\ School of Telecommunications Engineering, University of Valladolid, Valladolid 47011, Spain \\ ${ }^{3}$ Civil Engineering Department, Superior Technical School, University of Burgos, Burgos 09006, Spain \\ Correspondence should be addressed to Lara del Val; lvalpue@eii.uva.es
}

Received 9 January 2020; Revised 1 July 2020; Accepted 23 July 2020; Published 10 August 2020

Academic Editor: Rui Moreira

Copyright (C) 2020 Lara del Val et al. This is an open access article distributed under the Creative Commons Attribution License, which permits unrestricted use, distribution, and reproduction in any medium, provided the original work is properly cited.

\begin{abstract}
This paper presents the comparison of three methodologies to detect if some fans in a matrix are not working properly. These methodologies are based on detecting fan failures by analysing acoustic images of the fan matrix, obtained using a planar array of MEMS microphones. Geometrical parameters of these acoustic images for different frequencies are then used to train a support vector machine (SVM) classifier, in order to detect the fan failures. One of the methodologies is based on the detection of the faulty fan in the matrix, under the hypothesis that only one fan can fail. Other methodology is based on the detection of the specific working situation of the matrix. And finally, the third methodology that is compared is based on determining individually if each of the fans of the matrix is working properly or not. The comparison shows that this third methodology is the most reliable.
\end{abstract}

\section{Introduction}

In recent years, techniques for obtaining acoustic images have been developed greatly and rapidly. At present, acoustic images are associated with a wide variety of applications [1], such as nondestructive testing of materials, medical imaging, underwater imaging, SONAR (SOund NAvigation and Ranging), and geophysical exploration. This work is related with one of these applications of acoustical imaging, obtaining acoustic images of machinery to be used in condition monitoring and fault detection tasks.

Fault detection lies in determining failures in machine structural components or abnormal behaviours of a system [2]. Condition monitoring is the process of monitoring a condition parameter in machinery (vibration and temperature), in order to identify a significant change, which is indicative of a fault. The use of condition monitoring allows maintenance to be scheduled [3] or other actions to be taken to prevent consequential damages and avoid its consequences, as a major failure.
One of the classic approaches for machinery condition monitoring is based on making periodic vibration measurements of the equipment and then comparing them to known healthy/damaged data to assess the health status of the machine [4-10]. Sometimes, vibrational measurements need a sensor mounted on the machine, as accelerometers, and this presence can imply disturbances on the machine response and performance. As vibrational responses are related to acoustic emissions, one possible solution to this problem is the analysis of the related acoustic responses, instead of the vibrational ones. As sound field contains abundant information related to fault patterns, acoustic-based diagnosis with noncontact measurement is an option to take into account [11-15] or combined with vibrational information [16-18]. There are also some studies that show methodologies that classify machinery failures by analysing acoustic emissions [19-21]. Particularly, there are many examples [22-25] of the use of microphone arrays in acoustic imaging systems to measure this acoustic field. Actually, arrays of MEMS microphones are specially designed for acoustical imaging, and there are also examples of the use of microphone arrays to detect failures on machinery $[26,27]$. 
An array is an arranged set of identical sensors, fed in a specific manner. The array beampattern can be controlled by modifying the geometry of the array (linear and planar), the sensor spacing, the response, the amplitude, and phase excitation of each sensor [28]. By using beamforming techniques [29], the array beampattern can be electronically steered to different spatial positions, allowing spatial filtering, i.e., the discrimination of acoustic sources on the basis of their position.

The acronym MEMS (microelectromechanical system) refers to mechanical systems with a dimension smaller than $1 \mathrm{~mm}$, manufactured with tools and technology arising from the integrated circuits (ICs) field, and used for the miniaturization of mechanical sensors [30]. The application of MEMS technology to acoustic sensors [31] has allowed the development of high-quality microphones with high SNR (signal-to-noise ratio), low power consumption, and high sensitivity.

Due to the high diversity of applications of arrays of MEMS microphones, the authors of this paper are working on widening these uses to other fields. The authors already had experience in the design and development of acoustic arrays to be used in surveillance systems [32], in ambientassisted living [33], in an identification system based on acoustic biometry [34-36], or in a high-resolution virtual array [37]. The works [38-41] shown in this paper present two novel contributions: (i) failure detection and condition monitoring of a fan matrix, based on acoustic images and (ii) the use of arrays of MEMS microphones to obtain these acoustic images. These acoustic images are obtained by using the modular and reconfigurable system [42] design by the authors, which is based on a planar array module of $8 \times 8$ MEMS microphones.

A fan matrix, fan array, or fan wall is a system formed by several fans located on a surface, working together in order to improve the performance of one alone large fan with lower power consumption. Any type of application that requires specific temperature conditions is a candidate for a fan matrix.

An analysis of the systems which uses fans matrices reveals that they do not have a subsystem to control if any of the fans that compose the matrix is down or is not working properly. It would be very useful to detect these kinds of situations. The authors have developed a novel fault diagnosis methodology to detect faulty behaviours on the fans included in a matrix. This method is based on the analysis and classification of the acoustic images, obtained from the fan matrix, by means of using machine learning techniques.

On the first step of this research [38], a previous analysis of the viability of using an array of $8 \times 8$ MEMS microphones to obtain acoustic images of fan matrices was carried out, obtaining positive results. In the subsequent research work [39] carried out by the authors, these obtained acoustic images of a matrix of fans were used to detect operation failures on some fans. Promising results showed that geometric parameters of these acoustic images, obtained using the planar array of MEMS microphones, could be used to detect if the fans are or are not working properly. The failure detection methodology shown in this paper is based on a support vector machine (SVM) classifier [43] that uses these geometrical parameters of the acoustic images of the fan matrix, obtained using a $16 \times 16$ planar array of MEMS microphones working at different frequencies.

On the basis of these results, this work analyses the behaviour of different detection methodologies based on detection if any of the fans of a matrix is not working properly. These methodologies are based on geometrical parameters of the acoustic images of the fan matrix and in SVM classifiers.

\section{Materials and Methods}

2.1. Hardware Setup. An acquisition and processing system, previously developed by the authors [42], has been used in this work. This system is based on a 2D array of MEMS microphones. In this work, as in a previous one [41], the acoustic images acquisition system is composed of 4 uniform planar arrays (UPA) modules of $8 \times 82.125 \mathrm{~cm}$ uniformly spaced digital MEMS microphones connected to a myRIO platform, as shown in Figure 1(a). Thus, the acoustic signals acquisition is performed by a $16 \times 16$ array, as shown in Figure 1(b).

After the acquisition of the acoustic signals by the MEMS microphones of the array, they are processed using deinterlacing, decimation, and filtering techniques, in order to generate the acoustic images using wideband beamforming. A set of $N \times N$ steering directions are defined, and the beamformer outputs are assessed for each of these steering directions. The acoustic images generated are then displayed and stored in the system. The algorithms implemented in the system are shown in Figure 2.

This work is focused on obtaining acoustic images of a $3 \times 3$ fan matrix, shown in Figure 3 . Each of the fans used to build the fan matrix is a Foxconn D90SM-12 3-Pin with 7 blades, as in the previous study [41]. As it is shown in Figure 3, the fans of the matrix are controlled by a Kkmoon 8-channel relay interface board, which can be controlled by a microcontroller. The interface board of the control system allows turning on and off the fans of the matrix independently.

For the tests, the fan matrix is placed $50 \mathrm{~cm}$ opposite the $16 \times 16$ MEMS array, inside a $5 \mathrm{~m} \times 3 \mathrm{~m} \times 2.5 \mathrm{~m}$ anechoic chamber, as shown in Figure 4.

\subsection{Previous Analysis of the Fan Matrix Acoustic Images.}

The first step of this work was an analysis of the acoustic signals received by the microphones of the array, in order to characterize the noise generated by the fans of the matrix and to select the frequencies used to obtain the acoustic images of the fan matrix. As each fan has 7 blades and it rotates at $3500 \mathrm{rpm}$, its noise has harmonics at $400 \mathrm{~Hz}$ and its multiples. In this previous study [40], it was decided to work with the acoustic images at the harmonic frequencies between $400 \mathrm{~Hz}$ and $4000 \mathrm{~Hz}$ (10 different working frequencies). It was also observed that fan noise is not stationary or periodic. Working with nonstationary signals is complex; so, working with averaged signals is an easier option. The 


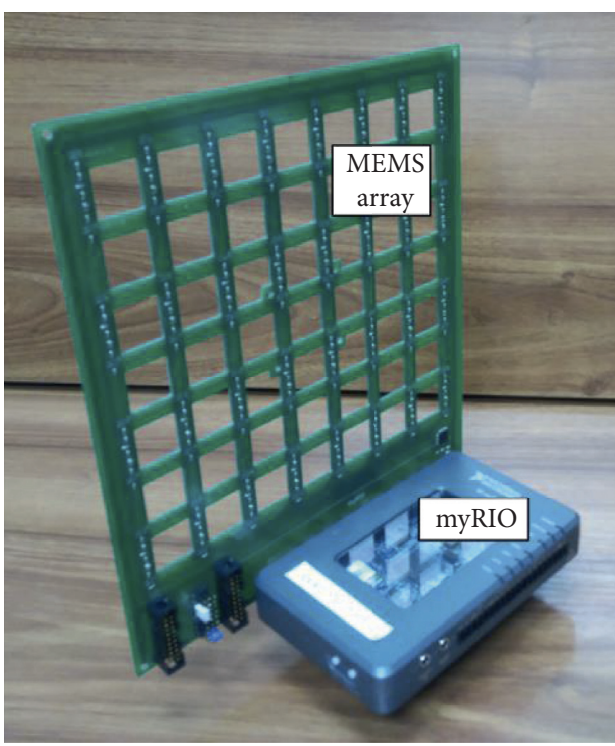

(a)

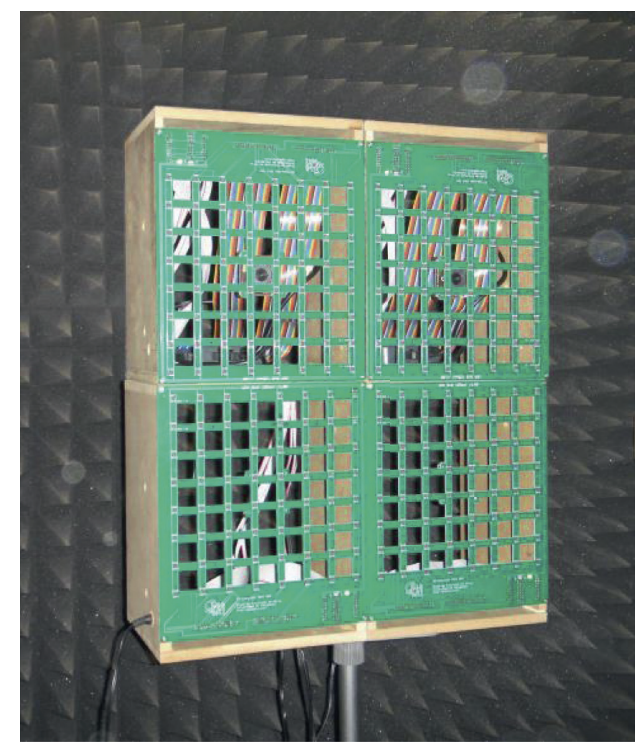

(b)

Figure 1: (a) Array module with myRIO and MEMS array board. (b) Complete acoustic acquisition system.

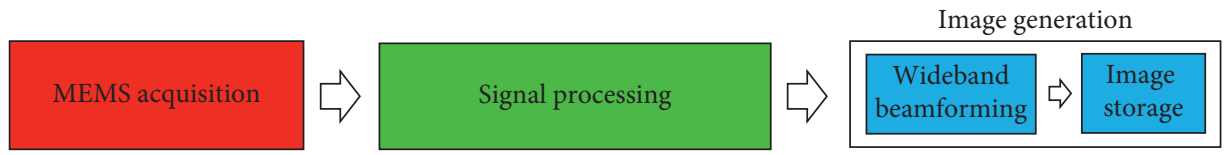

FIGURE 2: Software algorithms diagram.

authors decided to capture 1000 acoustic images for each specific test, in order to obtain these averaged acoustic images for each fan matrix working situation and analyse them to obtain the averaged behaviour of the fan matrix noise.

Preliminary tests [38], which were carried out by the authors, simulated a faulty fan matrix, i.e., with some fans working and the others damaged. The objective of these tests was to analyse the acoustic images of the fan matrix in these certain circumstances. The first row in Figure 4 shows captures of the interface of the relay board where a green square represents a working fan, and a red square represents a faulty one. The second row in Figure 4 shows the acoustic images of three different faulty fan matrix situations:

(a) Only the fans on the corners of the matrix are working (left column)

(b) The fans of the middle column of the matrix are faulty (middle column)

(c) The fans of the middle row of the matrix are faulty (right column)

Analysing these images, it can be observed that if several fans of the matrix are not running, i.e., are faulty, the acoustic image reveals this effect in some way.

In Figure 5(a), it can be observed that the acoustic image shows sound only in the positions of the fans that are working. In Figures 5(b) and 5(c), the acoustic images show an absence of sound in the positions of the faulty fans, on the middle column in Figure 5(b) and on the middle row in Figure 5(c). Figure 5 confirms that the acoustic images of fan matrices reveal the healthy/faulty character of the working matrix. This fact is the basis of the work presented on this paper.

\section{Results and Discussion}

This section describes the 3 fault detection methodologies that are going to be compared. All these methodologies are found on a machine learning algorithm, based on a linear support vector machine (SVM). Geometrical parameters of the acoustic images were used in this machine learning algorithm. These geometrical parameters were the value and the position (azimuth and elevation) of the maxima of the acoustic images for each of the 10 selected working frequencies, defined in Section 2.2. So, the SVM algorithms have worked with 30 parameters.

Figure 6 shows a block diagram of the different steps that form the methodologies that are compared in this work. The differences between the methodologies are based, mainly, on the definition of the SVM classification.

3.1. One Faulty Fan Detection Methodology. A set of tests $[40,41]$ was carried out on previous studies, simulating a matrix with only one faulty fan, and the corresponding acoustic images were obtained. With these tests, the authors worked under the hypothesis that the fans of the matrix fail 


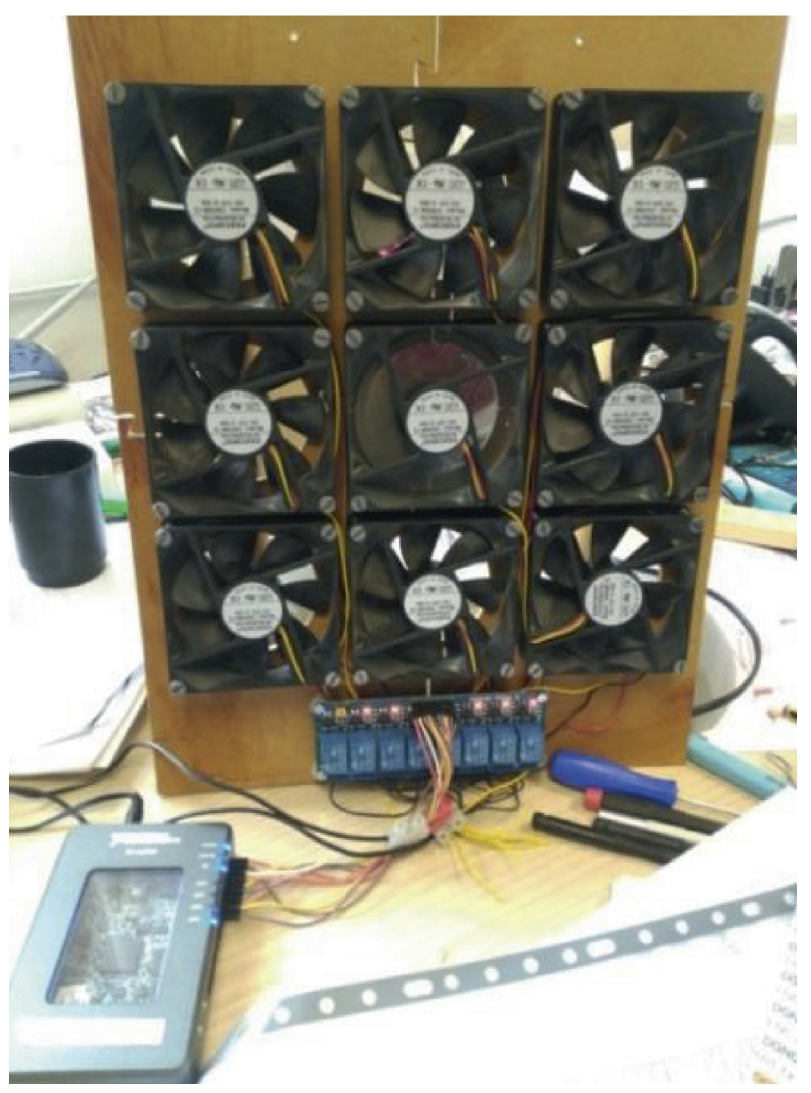

Figure 3: Test fan matrix.

one by one. The problem with these images was that they do not reveal, at a first glance, if there is a faulty fan, as the images in Figure 4. But, in fact, they included information about the faulty character of the matrix, although this information is not visible.

In this methodology, the SVM algorithm was used to detect the faulty fan position. The employed SVM worked with 10 different classes: 1 class represented a healthy matrix (all working fans), and the other 9 classes represented the 9 possible one faulty fan situations. As it was pointed previously, the SVM algorithm used 30 geometrical parameters of the acoustic images. In these tests, it was noticed that if one fan failed, the maximum position and value of the acoustic image changed. One of these effects is shown in Figure 5, which shows the maximum positions of the acoustic images, for the 10 defined working frequencies of the 9 one faulty fan situations [40]. In Figure 7, the real position of the corresponding faulty fan is shown as a cross.

The obtained accuracy rate by the SVM algorithm, trying to detect the faulty fan, was $95.6 \%$. This result showed that the purposed methodology was reliable when one fan of the matrix failed because it was accurate for detecting the position of the faulty fan.

Although it is really unusual that more than one fan fails at the same time, a set of tests was carried out in order to study if this defined methodology was robust enough in the presence of unexpected situations, i.e., if the number of

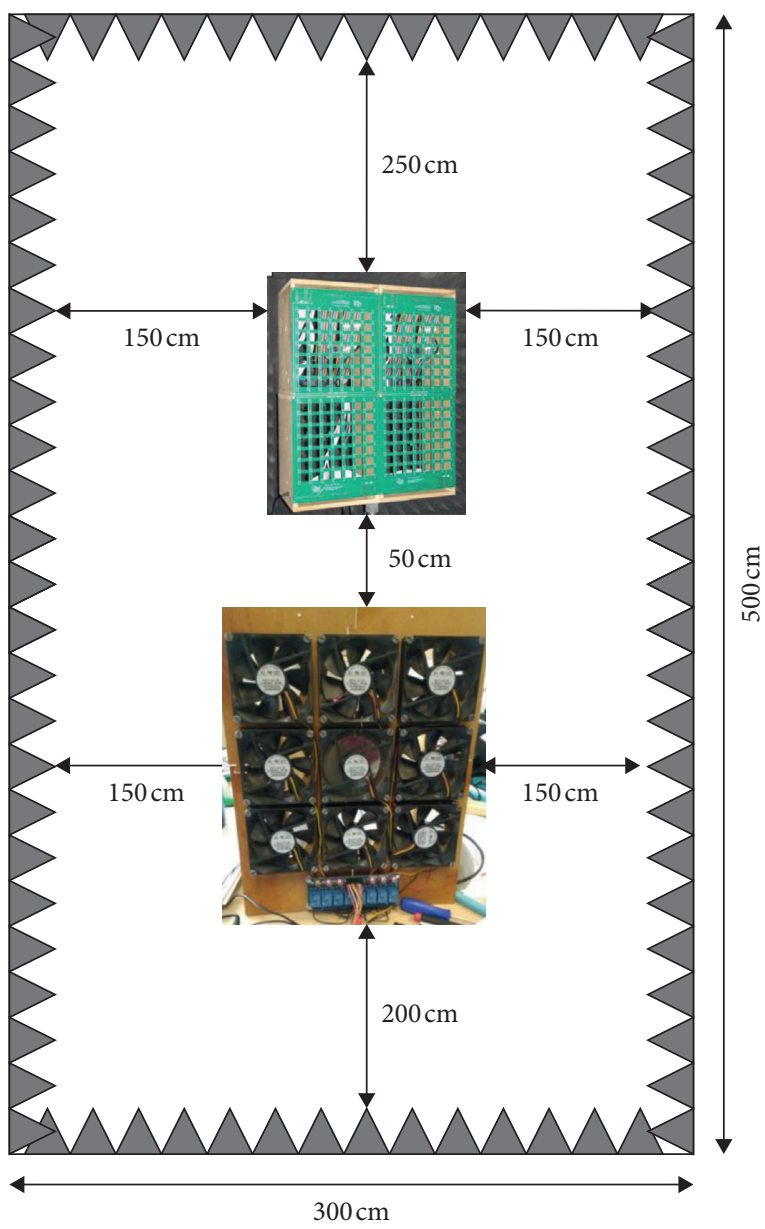

FigURE 4: Experimental setup block diagram.

faulty fans in the matrix increased [41]. In these new tests, the SVM classifier was trained with the geometrical parameters of the acoustic images of the one faulty fan working situations, and after that, it was validated with the geometrical parameters of the acoustic images of different two faulty fans working situations.

The objective of these tests was to analyse if the SVM algorithm, trained to detect only one faulty fan, was robust enough to detect any of the two faulty fans of the working situations. With a robust methodology, if one of the two faulty fans was detected, it would be repaired or replaced. After that, when the matrix began to run again, the other faulty fan would be detected and replaced. In these tests, if the algorithm did not detect any of the two faulty fans, it was considered that the algorithm failed. The results obtained in these tests showed that if the distance between the two faulty fans increased, the accuracy of the algorithm to detect that one of them failed or decreased. Table 1 shows the accuracy rates of the algorithm, trained to detect only one faulty fan, and validated under only one faulty fan situations and under different two faulty fans working situations. In these tests, although in most cases the SVM classifier was not able to correctly identify which were the faulty fans, it always alerted if the matrix was not working properly. 


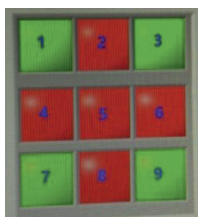

Working fan

Faulty fan

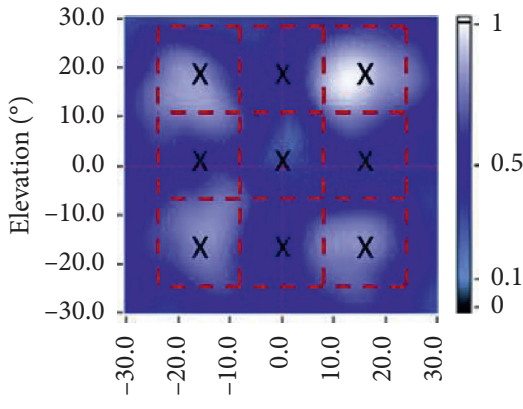

Azimuth $\left(^{\circ}\right)$

(a)

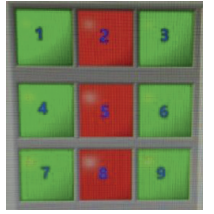

Working fan

Faulty fan

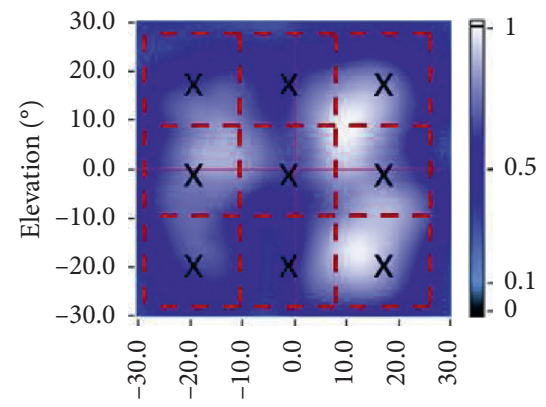

Azimuth $\left(^{\circ}\right)$

(b)

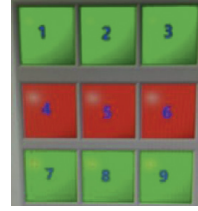

Working fan

Faulty fan

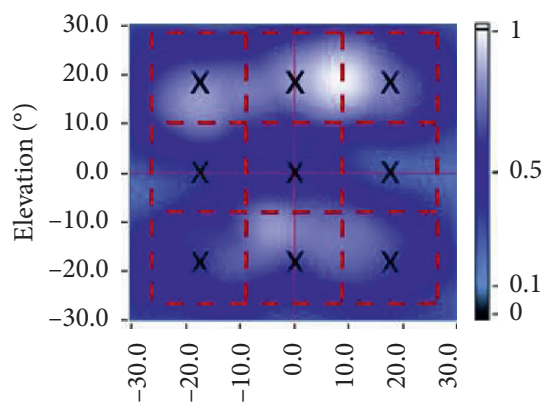

Azimuth $\left(^{\circ}\right)$

(c)

FIGURE 5: Acoustic images of faulty fan matrices: (a) only the fans on the corners are working, (b) the fans of the middle column are faulty, and (c) the fans on the middle row are faulty.

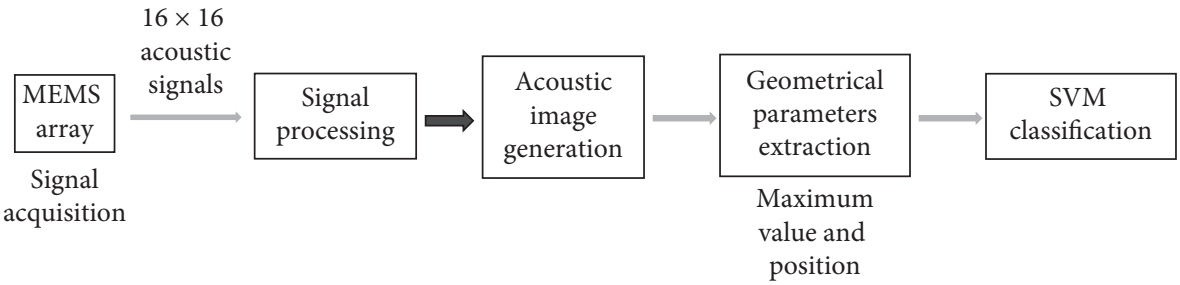

FIGURE 6: Block diagram of the compared methodologies.

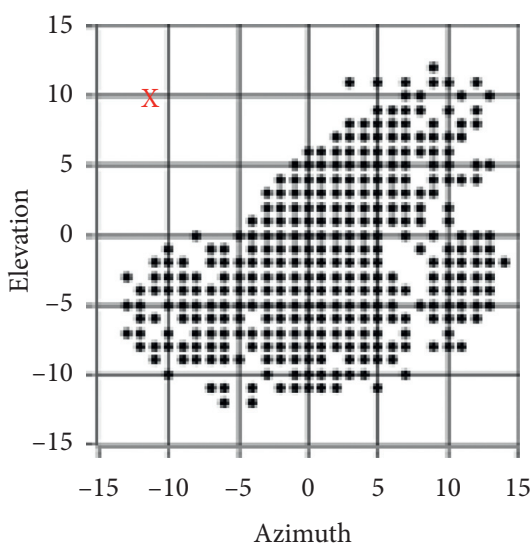

(a)

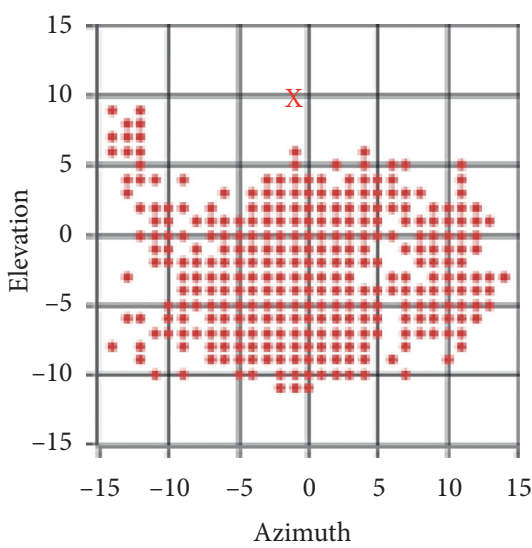

(b)

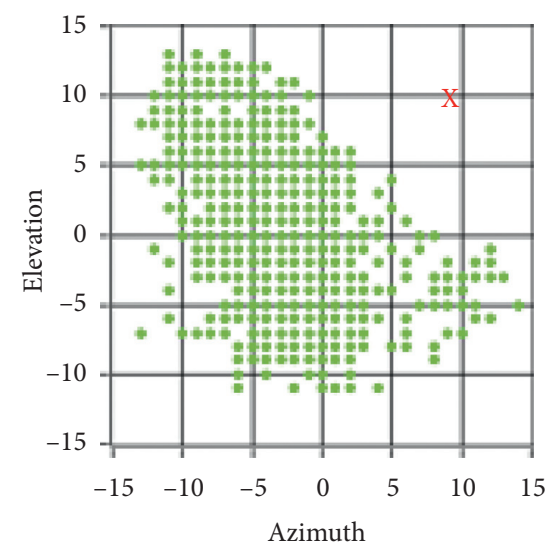

(c)

FIgURE 7: Continued. 


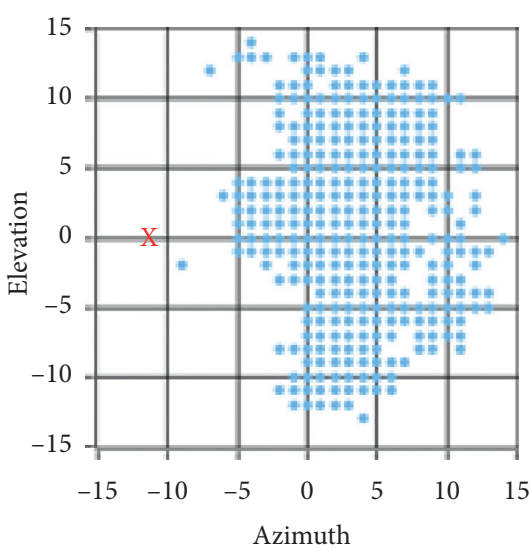

(d)

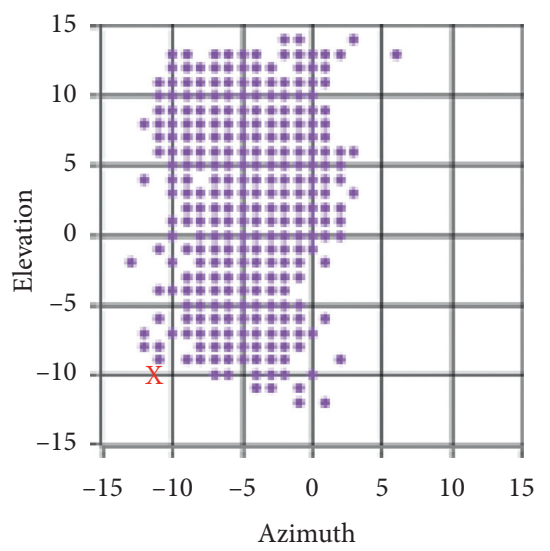

(g)

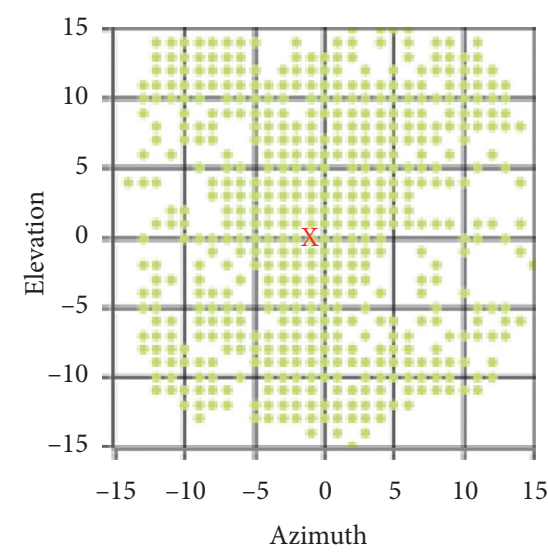

(e)

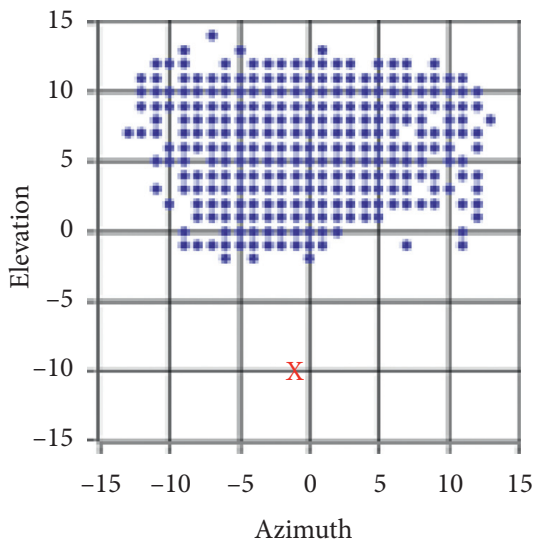

(h)

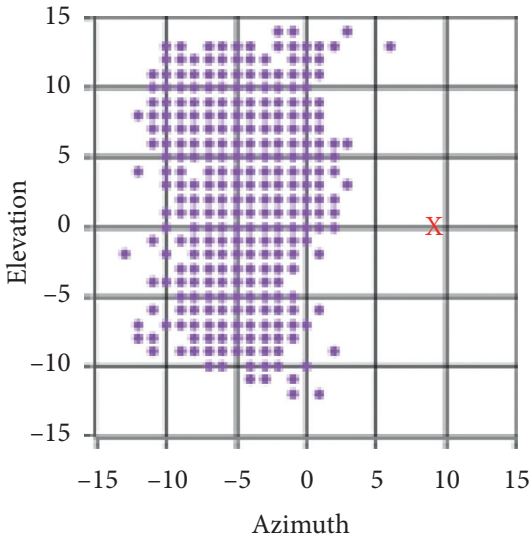

(f)

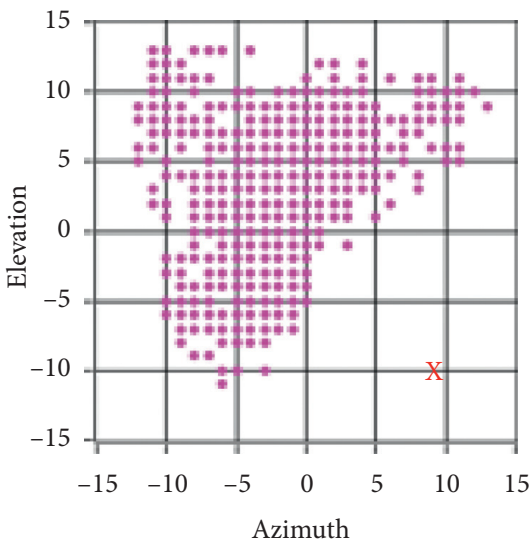

(i)

FIgURE 7: Maximum positions of the acoustic images of fan matrix with one faulty fan (the position of this faulty fan is represented with a cross). (a) Fan 1. (b) Fan 2. (c) Fan 3. (d) Fan 4. (e) Fan 5. (f) Fan 6. (g) Fan 7. (h) Fan 8. (i) Fan 9.

TABLE 1: SVM accuracy rates of the robustness tests for the one faulty fan detection methodology based on training the SVM with one faulty fan parameters.

One faulty fan tests

(1) Faultyfaulty fan validation

(2) Faultyfaulty fans validation

Case A: horizontal/vertical step distance (no central fan)

Case B: horizontal/vertical step distance (with central fan)

Case C: diagonal step distance

Case D: knight movement distance

Case E: row/column distance

Case F: diagonal distance
Accuracy rate (\%)

95.6

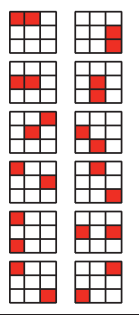

48.3

44.1

23.5

14.7

14.2

10.9
3.2. Working Situation Detection Methodology. As the one faulty fan detection methodology is not considered to be robust enough under unexpected situations (more than one faulty fan), the following implemented tests have been aimed to train the SVM algorithm to be able to detect any of the 512 different working situations of the fan matrix $\left(512=2^{9}\right.$, since the matrix is composed of 9 fans, each one with 29 different working states: working/healthy or faulty). These 512 working situations are considered from the perfectly working situation (all 9 working fans) to the impossible working situation (all 9 faulty fans), including all the remaining ones (one faulty fan, two faulty fans, and three faulty fans). So, this SVM algorithm works with 512 classes and the same 30 geometrical parameters than in the previous methodology used.

The obtained accuracy rate by the SVM algorithm in these tests has been $45.3 \%$. This result shows that this purposed methodology is not reliable. Training the SVM algorithm in order to be able to discriminate among 512 different classes is not a reliable option. The employed data 
TABLE 2: SVM accuracy rates of each classifier related to one of the fans of the matrix.

\begin{tabular}{lc}
\hline SVM classifier & Accuracy rate (\%) \\
\hline Fan 1 & 99.4 \\
Fan 2 & 91.0 \\
Fan 3 & 98.0 \\
Fan 4 & 91.4 \\
Fan 5 & 95.4 \\
Fan 6 & 98.9 \\
Fan 7 & 90.7 \\
Fan 8 & 91.6 \\
Fan 9 & 98.5 \\
\hline
\end{tabular}

(30 parameters for each test) is not enough to discriminate among so many classes (512 different working situations).

3.3. Individual Faulty Fan Detection Methodology. So, the next idea has been the definition of a methodology based on 9 independent SVM classifiers, each one related with a specific fan of the matrix. Each classifier has been trained to detect if the corresponding fan is working or not, that is, to detect 2 different classes. Again the methodology uses 30 geometrical parameters to detect if the matrix is not working properly. Table 2 shows the accuracy rates obtained by each of the 9 SVM classifiers related to a specific fan of the matrix. These results show that the accuracy of each of the SVM classifiers is high, given that all the accuracy rates are higher than $90 \%$.

The problem with this methodology is that despite the individual accuracies of each of the 9 SVM classifiers being high, the global accuracy of this methodology is not so high. The global accuracy rate of this methodology, with the 9 SVM classifiers working properly at the same time (all of them detecting if the corresponding fan is working properly or not), falls to a $65.7 \%$ value. It seems that detection failures of the SVM classifiers do not match between them. This global value takes into account all the possible matrix failures, the 512 possibilities.

It has been previously noticed that a failure situation in the matrix with more than two faulty fans is not probable. So, a group of tests is performed to assess the accuracy of this individual faulty fan detection methodology when there are only two faulty fans that have been carried out. The obtained global accuracy of the methodology for all these working situations is $87.9 \%$, and it is shown in Table 3 . If this value is compared with the accuracy values obtained with the one faulty fan detection methodology validated with two faulty fan working situations, shown in Table 1, this new methodology improves the detection accuracy. Previous values on Table 1 showed accuracy rates between $10.9 \%$ and $48.3 \%$, which are highly lower than the $87.9 \%$ accuracy rate obtained in these new tests.

In order to be able to compare the shown methodologies, the same two faulty fans working situations as those shown in Table 1 have been tested. These working situations consider different two faulty fans separations or positions: (i) Case A: two fans located on both ends of the largest diagonal of the matrix

(ii) Case B: two fans located on both ends of one row or one column of the matrix

(iii) Case C: two fans located on both ends of the " $\mathrm{L}$ shaped" (Knight) movement in chess

(iv) Case D: two fans separated one diagonal step

(v) Case E: one fan in the centre of the matrix, and the other one separated one vertical/horizontal step

(vi) Case F: two fans separated one vertical/horizontal step, and none is in the centre of the matrix

The obtained accuracy rates for the different tested cases are shown in Table 3. It can be observed that this methodology is robust enough to detect the corresponding two fans that are not working properly. In these cases, the obtained accuracy rates show high values between $84.3 \%$ and $92.5 \%$.

\section{Discussion}

Three different methodologies to detect faulty fans in a fan matrix have been defined. These methodologies are based on the maxima positions and values of the acoustic images obtained for the fan matrix at different working frequencies. A summary of these methodologies is shown in Table 4.

The first defined methodology is based on detecting one faulty fan. This methodology is highly accurate if only one fan is not working properly, but it is not robust enough under unexpected situations. If one more fan fails, the accuracy rate of the methodology in detecting one of the two faulty fans fails to values between $10.6 \%$ and $48.3 \%$.

The second methodology is based on detecting the specific working situation of the fan matrix. As the matrix has 9 fans, 512 different working situations can be defined. The accuracy rate of this methodology is $45.3 \%$. This value shows that, as for the previous case, this methodology is not robust enough either.

The last implemented methodology is based on detecting if each of the fans is or not working properly. This methodology uses 9 SVM classifiers, instead of only one, but each classifier is trained to distinguish between only two classes (a faulty fan or a healthy fan). The individual accuracy rates of the SVM classifiers show high values between $90.7 \%$ and 99.4\%. This methodology is not accurate enough to distinguish a matrix failure among its 512 possible faulty situations. In this case, this methodology shows a $65.7 \%$ accuracy rate value. Although it is not a high accuracy value, it is higher than the one obtained for the methodology based on detecting the working situation of the fan matrix. But it is not a problem because the probability of having a fan matrix with more than two faulty fans is not high. Under this consideration, this methodology is a proper option, given that it shows high accuracy rate values in detecting faulty fans under two faulty fan working situations. In these cases, the methodology shows accuracy rate values between $84.3 \%$ and $92.5 \%$. 
TABLE 3: SVM accuracy rates of the individual faulty fan detection methodology with two faulty fans working situation of the matrix.

\begin{tabular}{ll}
\hline \multicolumn{1}{c}{ Individual faulty fan detection methodology: two faulty fans tests } & Accuracy rate (\%) \\
\hline Global & 87.9 \\
Two faulty fans separation \\
Case A: horizontal/vertical step distance (no central fan) \\
Case B: horizontal/vertical step distance (with central fan) \\
Case C: diagonal step distance \\
Case D: knight movement distance \\
Case E: row/column distance \\
Case F: diagonal distance
\end{tabular}

TABLE 4: SVM accuracy rates of the detection methodologies.

\begin{tabular}{|c|c|c|c|}
\hline \multirow[b]{2}{*}{ Methodology } & \multicolumn{2}{|c|}{ SVM } & \multirow{2}{*}{$\begin{array}{c}\text { Accuracy rate } \\
(\%)\end{array}$} \\
\hline & $\begin{array}{c}\# \\
\text { classifiers }\end{array}$ & $\begin{array}{c}\# \\
\text { classes }\end{array}$ & \\
\hline \multicolumn{4}{|c|}{ One faulty fan detection } \\
\hline $\begin{array}{l}\text { One faulty fan } \\
\text { validation }\end{array}$ & 1 & 10 & 95.6 \\
\hline $\begin{array}{l}\text { Two faulty fan } \\
\text { validation }\end{array}$ & & & $(10.6-48.3)$ \\
\hline \multicolumn{4}{|c|}{ Working situation detection } \\
\hline $\begin{array}{l}512 \text { working } \\
\text { situations }\end{array}$ & 1 & 512 & 45.3 \\
\hline \multicolumn{4}{|c|}{ Individual faulty fan detection } \\
\hline $\begin{array}{l}\text { Individual SVM } \\
\text { classifier }\end{array}$ & 9 & 2 & $(90.7-99.4)$ \\
\hline $\begin{array}{l}512 \text { working } \\
\text { situations }\end{array}$ & & & 65.7 \\
\hline $\begin{array}{l}\text { Two faulty fans } \\
\text { situations }\end{array}$ & & & 87.9 \\
\hline
\end{tabular}

\section{Conclusions}

This paper shows the comparison of three fault detection methodologies developed to identify if the fans in a matrix are not working properly. These methodologies are based on geometrical parameters of the acoustic images of the fan matrix and in support vector machine algorithms.

The most promising methodology is based on 9 SVM classifiers, each one related to a specific fan of the matrix. Each individual classifier detects if the corresponding fan is or not working. These high accuracy rate values shown by this methodology balance out the complexity increment related to using a SVM classifier for each fan of the matrix, instead of only one, as in the other defined methodologies.

It could be pointed that the tests carried out in this study must be widen with other tests including background noise or even objects near the assembly, in order to create surfaces where the sound generated by the fans could be reflected. These tests would simulate a more real operation situation because an operative fan matrix is not isolated of the surroundings nor even placed inside an anechoic chamber.

As near future work, a new methodology which combines the information of the maxima and other features or geometrical parameters of the acoustic images, such as the centroids or the energy, could be defined. In this case, if the
SVM algorithm gets more information to discriminate between the different working situations, it is expected to be able to improve its accuracy rate. Also as a midterm future work, the extension of the methodology is to be able to detect/classify/identify specific fan failures such as not only to detect if the fans are working or not but also to detect the reason why the fan is not working properly, that is, if it is not rotating at the right speed, if some of the blades are broken, and if the shaft is misaligned. So, the next step in this research is the fan future work which could be the comparison of this extended methodology with other methodologies based on rotating machines that classifies specific failures, such as MSAF methodologies.

\section{Data Availability}

The numeric data used to support the findings of this study are available from the corresponding author upon request.

\section{Conflicts of Interest}

The authors declare that there are no conflicts of interest regarding the publication of this paper.

\section{Acknowledgments}

This research was funded by MINECO/FEDER (EU) (SAM TEC 2015-68170-R).

\section{References}

[1] W. Siong, Acoustic Imaging: Techniques and Applications for Engineers, John Wiley \& Sons, Chichester, UK, 2012.

[2] V. Reppa and A. Tzes, "Fault detection and diagnosis based on parameter set estimation," IET Control Theory and Applications, vol. 5, no. 1, pp. 69-83, 2009.

[3] A. K. S. Jardine, D. Lin, and D. Banjevic, "A review on machinery diagnostics and prognostics implementing condition-based maintenance," Mechanical Systems and Signal Processing, vol. 20, no. 7, pp. 1483-1510, 2006.

[4] R. Burdzik, M. Cao, A. Katunin, and S. Ručevskis, "Vibrationbased damage identification and condition monitoring in mechanical structures and components," Shock and Vibration, vol. 2018, Article ID 6750937, 2 pages, 2018.

[5] G. Dalpiaz, R. Rubini, G. D’Elia et al., “Advances in condition monitoring of machinery in non-stationary operations," in Proceedings of the 2013 International Conference Condition 
Monitoring of Machinery in Non-Stationary Operations, Ferrara, Italy, May 2013.

[6] A. Martini, M. Troncossi, and A. Rivola, "Automatic leak detection in buried plastic pipes of water supply networks by means of vibration measurements," Shock and Vibration, vol. 2015, Article ID 165304, 13 pages, 2015.

[7] G. Carvalho, R. Dalledone, A. Chaves, and M. Feiertag, "Experimental aspects in the vibration-based condition monitoring of large hydrogenerators," International Journal of Rotating Machinery, vol. 2017, Article ID 1805051, 14 pages, 2017.

[8] A. Kowalska-Koczwara, F. Pachla, P. Stecz et al., "Vibrationbased damage identification and condition monitoring of metro trains: warsaw metro case study," Shock and Vibration, vol. 2018, Article ID 8475684, 14 pages, 2018.

[9] P. Gangsar and R. Tiwari, "Signal based condition monitoring techniques for fault detection and diagnosis of induction motors: a state-of-the-art review," Mechanical Systems and Signal Processing, vol. 144, Article ID 106908, 2020.

[10] Y. Xu, D. Zhen, J. X. Gu et al., "Autocorrelated Envelopes for early fault detection of rolling bearings," Mechanical Systems and Signal Processing, vol. 146, no. 1, Article ID 106990, 2021.

[11] H. Koruk and K. Y. Sanliturk, "Detection of air leakage into vacuum packages using acoustic measurements and estimation of defect size," Mechanical Systems and Signal Processing, vol. 114, pp. 528-538, 2019.

[12] A. Parey and A. Singh, "Gearbox fault diagnosis using acoustic signals, continuous wavelet transform and adaptive neurofuzzy inference system," Applied Acoustics, vol. 147, pp. 133-140, 2019.

[13] C. Beale, C. Niezrecki, and M. Inalpolat, "An adaptive wavelet packet denoising algorithm for enhanced active acoustic damage detection from wind turbine blades," Mechanical Systems and Signal Processing, vol. 142, Article ID 106754, 2020.

[14] E. Caso, A. Fernandez-del-Rincon, P. García, M. Iglesias, and F. Viadero, "Monitoring of misalignment in low speed geared shafts with acoustic emission sensors," Applied Acoustics, vol. 159, Article ID 107092, 2020.

[15] D. Zhang, M. Entezami, E. Stewart, C. Roberts, and D. Yu, "Adaptive fault feature extraction from wayside acoustic signals from train bearings," Journal of Sound and Vibration, vol. 425 , pp. 221-238, 2018.

[16] P. A. Delgado-Arredondo, D. Morinigo-Sotelo, R. A. OsornioRios, J. G. Avina-Cervantes, H. Rostro-Gonzalez, and R. d. J. Romero-Troncoso, "Methodology for fault detection in induction motors via sound and vibration signals," Mechanical Systems and Signal Processing, vol. 83, pp. 568-589, 2017.

[17] A. P. Patil, B. K. Mishra, and S. P. Harsha, "Vibration based modelling of acoustic emission of rolling element bearings," Journal of Sound and Vibration, vol. 468, Article ID 115117, 2020.

[18] Y. Liu, J. Han, Z. Xue, Y. Zhang, and Q. Yang, "Structural vibrations and acoustic radiation of blade-shafting-shell coupled system," Journal of Sound and Vibration, vol. 463, Article ID 114961, 2019.

[19] A. Glowacz, "Recognition of acoustic signals of commutator motors," Applied Sciences, vol. 8, no. 2630, pp. 1-21, 2018.

[20] A. Glowacz, "Fault detection of electric impact drills and coffee grinders using acoustic signals," Sensors, vol. 19, no. 269, pp. 1-22, 2019.

[21] A. Glowacz, "Acoustic fault analysis of three commutator motors," Mechanical Systems and Signal Processing, vol. 133, pp. 1-23, 2019.

[22] W. Lu, W. Jiang, G. Yuan, and L. Yan, "A gearbox fault diagnosis scheme based on near-field acoustic holography and spatial distribution features of sound field," Journal of Sound and Vibration, vol. 332, no. 10, pp. 2593-2610, 2013.

[23] P. Ravettan, J. Muract, and R. Burdisso, "Feasibility study of microphone phased array based machinery health monitoring," Mecánica Computacional, vol. 26, pp. 23-37, 2007.

[24] G. Herold and E. Sarradj, "Microphone array method for the characterization of rotating sound sources in axial fans," Noise Control Engineering Journal, vol. 63, no. 6, pp. 546-551, 2015.

[25] F. J. Zenger, G. Herold, S. Becker, and E. Sarradj, "Sound source localization on an axial fan at different operating points," Experiments in Fluids, vol. 57, no. 8, pp. 1-10, 2016.

[26] E. Cardenas Cabada, Q. Leclere, J. Antoni, and N. Hamzaoui, "Fault detection in rotating machines with beamforming: spatial visualization of diagnosis features," Mechanical Systems and Signal Processing, vol. 97, pp. 33-43, 2017.

[27] L. A. L. Janssen and I. López Arteaga, "Data processing and augmentation of acoustic array signals for fault detection with machine learning," Journal of Sound and Vibration, vol. 483, Article ID 115483, 2020.

[28] H. Van, Trees, Optimum Array Processing: Part IV of Detection, Estimation and Modulation Theory, John Wiley \& Sons, New York, NY, USA, 2002.

[29] B. D. Van Veen and K. M. Buckley, "Beamforming: a versatile approach to spatial filtering," IEEE ASSP Magazine, vol. 5, no. 2, pp. 4-24, 1988.

[30] S. Beeby, G. Ensell, M. Kraft, and N. White, MEMS Mechanical Sensors, Artech House Publishers, Norwood, MA, USA, 2004.

[31] P. R. Scheeper, A. G. H. Van der Donk, W. Olthuis, and P. Bergveld, "A review of silicon microphones," Sensors and Actuators A: Physical, vol. 44, no. 1, pp. 1-11, 1994.

[32] J. D. de Durán, J. J. V. Calvo, and A. I. Fuente, "Surveillance system based on data fusion from image and acoustic sensors," IEEE Aerospace and Electronic Systems Magazine, vol. 15 , no. 2 , pp. $9-16,2000$.

[33] J. J. Villacorta, M. I. Jiménez, L. del Val, and A. Izquierdo, "A configurable sensor network applied to ambient assisted living," Sensors, vol. 11, no. 11, pp. 10724-10737, 2011.

[34] A. Izquierdo, L. del Val, M. I. Jiménez, and J. J. Villacorta, "Performance evaluation of a biometric system based on acoustic images," Sensors, vol. 11, no. 10, pp. 9499-9519, 2011.

[35] A. Izquierdo, L. del Val, J. J. Villacorta, and M. Raboso, "Optimization of a biometric system based on acoustic images," The Scientific World Journal, vol. 2014, Article ID 780835, 13 pages, 2014.

[36] L. del Val, A. Izquierdo-Fuente, J. Villacorta, and M. Raboso, "Acoustic biometric system based on preprocessing techniques and linear support vector machines," Sensors, vol. 15, no. 6, pp. 14241-14260, 2015.

[37] A. Izquierdo, J. J. Villacorta, L. del Val, L. Suárez, and D. Suárez, "Implementation of a virtual microphone array to obtain high resolution acoustic images," Sensors, vol. 18, no. 1, pp. 25-37, 2018.

[38] L. del Val, A. Izquierdo, J. J. Villacorta, and L. Suárez, "Using a planar array of MEMS microphones to obtain acoustic images of a fan matrix," Journal of Sensors, vol. 2017, Article ID 3209142, 10 pages, 2017.

[39] L. del Val, A. Izquierdo, J. J. Villacorta, L. Suárez, and M. Herráez, "First steps on fan matrix condition monitoring and fault diagnosis using an array of digital MEMS microphones," Proceedings of Meetings on Acoustics, vol. 30, pp. 1-10, Article ID 030014, 2017.

[40] L. del Val, A. Izquierdo, J. J. Villacorta, L. Suárez, and M. Herráez, "Analysis of a maxima position estimator on 
acoustic images of a fan matrix for failure detection," in Proceedings of the Euronoise 2018 Conference, pp. 27-31, Crete, Greece, May 2018.

[41] L. del Val, A. Izquierdo, J. J. Villacorta, M. Herráez, and L. Suárez, "Fault detection methodology for a fan matrix based on SVM classification of acoustic images," in Proceedings of the 6th International Conference on Condition Monitoring of Machinery in Non-Stationary Operations, pp. 20-22, Santander, Spain, June 2018.

[42] A. Izquierdo, J. Villacorta, L. del Val Puente, and L. Suárez, "Design and evaluation of a scalable and reconfigurable multiplatform system for acoustic imaging," Sensors, vol. 16, no. 10, pp. 1671-1687, 2016.

[43] N. Cristianini and J. Shawe-Taylor, Support Vector Machines and other Kernel-Based Learning Methods, Cambridge University Press, Cambridge, UK, 1st edition, 2000. 